\title{
Metabolic engineering of Escherichia coli for the synthesis of polyhydroxyalkanoates using acetate as a main carbon source
}

Jing Chen ${ }^{1,2}$, Wei Li ${ }^{1,2}$, Zhao-Zhou Zhang ${ }^{2}$, Tian-Wei Tan² and Zheng-Jun Li ${ }^{1,2^{*}}$

\begin{abstract}
Background: High production cost of bioplastics polyhydroxyalkanoates (PHA) is a major obstacle to replace traditional petro-based plastics. To address the challenges, strategies towards upstream metabolic engineering and downstream fermentation optimizations have been continuously pursued. Given that the feedstocks especially carbon sources account up to a large portion of the production cost, it is of great importance to explore low cost substrates to manufacture PHA economically.
\end{abstract}

Results: Escherichia coli was metabolically engineered to synthesize poly-3-hydroxybutyrate (P3HB), poly(3-hydroxybutyrate-co-4-hydroxybutyrate) (P3HB4HB), and poly(3-hydroxybutyrate-co-3-hydroxyvalerate) (PHBV) using acetate as a main carbon source. Overexpression of phosphotransacetylase/acetate kinase pathway was shown to be an effective strategy for improving acetate assimilation and biopolymer production. The recombinant strain overexpressing phosphotransacetylase/acetate kinase and P3HB synthesis operon produced $1.27 \mathrm{~g} / \mathrm{L} \mathrm{P3HB}$ when grown on minimal medium supplemented with $10 \mathrm{~g} / \mathrm{L}$ yeast extract and $5 \mathrm{~g} / \mathrm{L}$ acetate in shake flask cultures. Further introduction succinate semialdehyde dehydrogenase, 4-hydroxybutyrate dehydrogenase, and CoA transferase lead to the accumulation of P3HB4HB, reaching a titer of $1.71 \mathrm{~g} / \mathrm{L}$ with a 4-hydroxybutyrate monomer content of $5.79 \mathrm{~mol} \%$. When $1 \mathrm{~g} / \mathrm{L}$ of a-ketoglutarate or citrate was added to the medium, P3HB4HB titer increased to 1.99 and $2.15 \mathrm{~g} / \mathrm{L}$, respectively. To achieve PHBV synthesis, acetate and propionate were simultaneously supplied and propionyl-CoA transferase was overexpressed to provide 3-hydroxyvalerate precursor. The resulting strain produced $0.33 \mathrm{~g} / \mathrm{L} \mathrm{PHBV}$ with a 3-hydroxyvalerate monomer content of $6.58 \mathrm{~mol} \%$. Further overexpression of propionate permease improved PHBV titer and 3-hydroxyvalerate monomer content to $1.09 \mathrm{~g} / \mathrm{L}$ and $10.37 \mathrm{~mol} \%$, respectively.

Conclusions: The application of acetate as carbon source for microbial fermentation could reduce the consumption of food and agro-based renewable bioresources for biorefineries. Our proposed metabolic engineering strategies illustrate the feasibility for producing polyhydroxyalkanoates using acetate as a main carbon source. Overall, as an abundant and renewable resource, acetate would be developed into a cost-effective feedstock to achieve low cost production of chemicals, materials, and biofuels.

Keywords: Acetate, Escherichia coli, Poly-3-hydroxybutyrate, Poly(3-hydroxybutyrate-co-4-hydroxybutyrate), Poly(3hydroxybutyrate-co-3-hydroxyvalerate)

\footnotetext{
*Correspondence: lizj@mail.buct.edu.cn

${ }^{1}$ Beijing Advanced Innovation Center for Soft Matter Science and Engineering, Beijing University of Chemical Technology, Mailbox 53,

No. 15 Beisanhuan Donglu, Chaoyang District, Beijing 100029, China

Full list of author information is available at the end of the article
} 


\section{Background}

Food and agro-based renewable bioresources, such as xylose [1], glucose [2], sucrose [3], and starch [4], are most widely used carbon feedstocks for the microbial production of polymers, biofuels and building block chemicals. Polyhydroxyalkanoates (PHA), the promising biodegradable polyesters which are produced by an extensive variety of microorganisms for carbon and energy storage purposes, are also synthesized from glucose as a main carbon source [5, 6]. Although considerable efforts have been devoted to decreasing the production cost of PHA to make it economically competitive, the present price of PHA is still not feasible to replace traditional petro-based plastics [7]. It is believed that the feedstocks especially carbon source accounts up to a large portion of the production cost. Therefore, it is important to develop low cost substrates to make PHA economically competitive [8]. In addition, the global food shortage and the price increases of agricultural products have made it difficult to rely on food-based materials as fermentation feedstocks, in addition to the ethical considerations involved with doing so. Consequently, the development of non-food based substrates, such as methanol [9], acetate [10], and syngas [11], is quickly becoming one of the most important research areas of industrial biotechnology.

Acetate is the second simplest carboxylic acid, and many microorganisms can utilize acetate as alternative carbon source for cell growth. The acetate assimilation species include Escherichia coli [12], Cryptococcus curvatus [13], Clostridium sp. [14], Halomonas boliviensis [15], and so on. Recent studies have demonstrated that acetate can be produced from low cost substrates via biochemical processes. For example, acetate is a major fermentation product during anaerobic digestion of organic wastes and syngas production by acetogenic bacteria Moorella thermoacetica from carbon dioxide [16, 17]. In addition, acetate is also widely existed as a byproduct from hydrolysis or pyrolysis of lignocellulosic biomass $[18,19]$. In this regard, compared with glucose, acetate would be a promising cost-effective carbon source suitable for microbial fermentation.

Oleaginous yeasts including C. curvatus [20] and Yarrowia lipolytica [21] have been reported to utilize waste acetate as a main carbon source to accumulate lipids. Although acetate is a notorious cell growth inhibitor for $E$. coli fermentation, recombinant $E$. coli strains were constructed to use acetate for the production of free fatty acids [22] and succinate [23]. Therefore, it is also quite interesting to investigate the possibility of producing PHA polymers from acetate.

In this study, the metabolic pathways of E. coli were engineered to produce poly-3-hydroxybutyrate
(P3HB), poly(3-hydroxybutyrate-co-4-hydroxybutyrate) (P3HB4HB), and poly(3-hydroxybutyrate-co-3-hydroxyvalerate) (PHBV) using acetate as a main carbon source (Fig. 1). We compared the effects of overexpressing phosphotransacetylase/acetate kinase and AMP-forming acetyl-CoA synthetase on acetate assimilation and $\mathrm{P} 3 \mathrm{HB}$ production. In addition, succinate semialdehyde dehydrogenase, 4-hydroxybutyrate dehydrogenase, and CoA transferase was overexpressed to construct P3HB4HB accumulation pathway. Propionyl-CoA transferase and propionate permease were introduced to provide 3-hydroxyvalerate precursor from propionate, which helped to achieve PHBV synthesis. Our results demonstrate that $\mathrm{P} 3 \mathrm{HB}, \mathrm{P} 3 \mathrm{HB} 4 \mathrm{HB}$, and $\mathrm{PHBV}$ can be effectively synthesized using acetate as a main carbon source, opening new approaches for the utilization of this cheap carbon source for the production of various high valueadded biomaterials.

\section{Methods}

\section{Bacterial strains and culture medium}

Escherichia coli JM109 was employed as the host for plasmid construction and $\mathrm{P} 3 \mathrm{HB}$ and PHBV production studies, while its succinate semialdehyde dehydrogenase mutant JM109SG [24] was used as the host for P3HB4HB production.

For construction of recombinants and plasmid amplification, E. coli strains were cultivated in Luria-Bertani (LB) medium at $37{ }^{\circ} \mathrm{C}$ and $200 \mathrm{rpm}$. LB medium contained $5 \mathrm{~g} / \mathrm{L}$ yeast extract, $10 \mathrm{~g} / \mathrm{L}$ Bacto tryptone, and $10 \mathrm{~g} / \mathrm{L} \mathrm{NaCl}$. To maintain plasmid stability, $100 \mu \mathrm{g} / \mathrm{mL}$ ampicillin and/or $50 \mu \mathrm{g} / \mathrm{mL}$ kanamycin was added to the media when required.

\section{Plasmid construction}

Strains and plasmids used in this study are listed in Table 1. Oligonucleotides used in this study are listed in Additional file 1: Table S1. Standard molecular cloning procedures or manufacturers' instructions were followed for plasmid construction. Q $5^{\circledR}$ High-Fidelity DNA Polymerase from New England Biolabs (Ipswich, MA, US) was employed for PCR reactions. Plasmid isolation and DNA purification kits were purchased from Biomed (Beijing, China). Restriction enzymes and DNA modifying enzymes were purchased from New England Biolabs.

The pta-ackA encoding phosphotransacetylase/acetate kinase and acs encoding acetyl-CoA synthetase were amplified from $E$. coli genomic DNA using primers pa1F/pa1R and acsF/acsR, respectively. The PCR products were purified and digested with HindIII/XbaI and inserted into the corresponding restriction sites of pEn to construct pEn-pta-ackA and pEn-acs, 


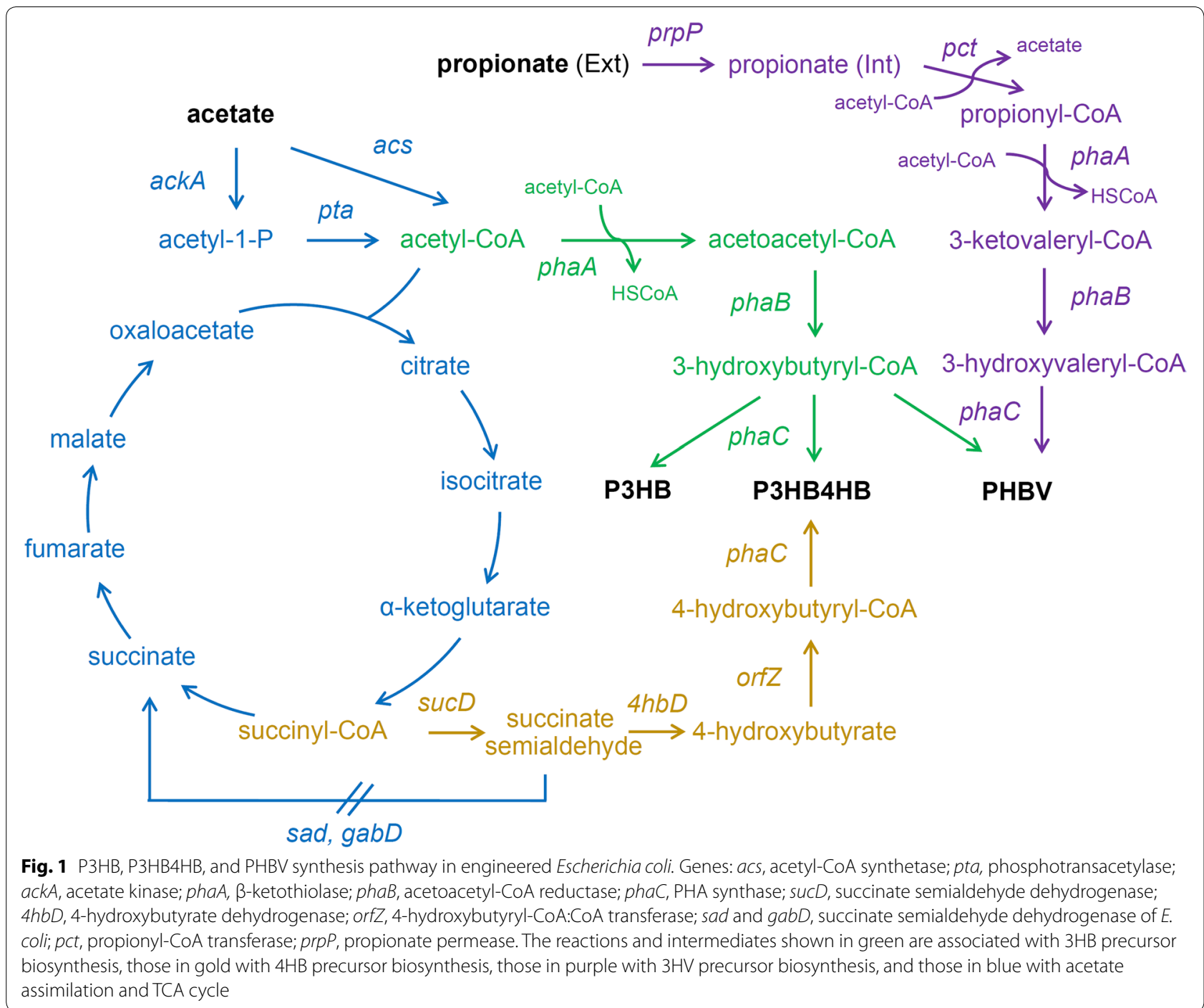

respectively. Moreover, $\mathrm{P}_{\mathrm{pdc}}$ promoter and gene coding sequence were removed from $\mathrm{pEn}$-derived plasmids and subcloned into kanamycin resistance carrying broad-host-range cloning vector pBBR1MCS-2 to generate pMCS-pta-ackA and pMCS-acs, respectively. Primers pa2F/pa2R were designed to amply pta-ackA from $\mathrm{pEn}$-pta-ackA. The PCR product was digested with $N h e \mathrm{I} / \mathrm{XhoI}$ and cloned into the $s u c D-4 h b D$ expression vector pMCSH5 cut with the same enzymes to form pMCSH5-pta-ackA. Primers prpPF/prpPR were designed to amply prpP encoding propionate permease from Ralstonia eutropha H16 genomic DNA. The PCR product was digested with BamHI/HindIII and cloned into $\mathrm{pEn}$ to generate $\mathrm{pEn}$-prpP. Subsequently, $\mathrm{P}_{\mathrm{pdc}}$-prpP was excised from pEn-prpP using SpeI/XhoI and cloned into pMCS-pta-ackA digested with NheI/XhoI to generate pMCS-pta-ackA-prpP.

\section{Growth conditions for biopolymer production}

To prepare the seed cultures, $10 \mu \mathrm{L}$ of glycerol stock was inoculated into $20 \mathrm{~mL}$ of $\mathrm{LB}$ medium and cultivated at $37{ }^{\circ} \mathrm{C}$ and $200 \mathrm{rpm}$ for $12 \mathrm{~h}$. For biopolymer producing shake flask studies, $4 \%(\mathrm{v} / \mathrm{v})$ of the seed culture was used to inoculate into $500 \mathrm{~mL}$ shake flasks containing $50 \mathrm{~mL}$ of medium and cultured at $37^{\circ} \mathrm{C}$ and $200 \mathrm{rpm}$ for $48 \mathrm{~h}$.

The plasmids including pBBR1MCS-2, pMCS-ptaackA, and pMCS-acs, each in combination with phaCAB expression plasmid pBHR68, were co-transformed into E. coli JM109 to generate $\mathrm{P} 3 \mathrm{HB}$ producing strains. Three different kinds of fermentation media were employed for P3HB production: $\mathrm{LB}$, terrific broth (TB), and minimal 
Table 1 Strains and plasmids used in this study

\begin{tabular}{|c|c|c|}
\hline Name & Relevant characteristics & References \\
\hline \multicolumn{3}{|l|}{ E. coli strains } \\
\hline JM109 & Wild type & {$[24]$} \\
\hline JM109SG & JM109 $\Delta s a d \Delta g a b D$ & {$[24]$} \\
\hline JM109 (pBHR68) & P3HB producing strain & This study \\
\hline JM109 (pBHR68 + pBBR1MCS-2) & P3HB producing strain & This study \\
\hline JM109 (pBHR68 + pMCS-pta-ackA) & P3HB producing strain & This study \\
\hline JM109 (pBHR68 + pMCS-acs) & P3HB producing strain & This study \\
\hline JM109SG (p68orfZ + pMCSH5) & P3HB4HB producing strain & This study \\
\hline JM109SG (p68orfZ + pMCSH5-pta-ackA) & P3HB4HB producing strain & This study \\
\hline JM109 (p68-pct-CAB + pBBR1MCS-2) & PHBV producing strain & This study \\
\hline JM109 (p68-pct-CAB + pMCS-pta-ackA) & PHBV producing strain & This study \\
\hline JM109 (p68-pct-CAB + pMCS-pta-ackA-prpP) & PHBV producing strain & This study \\
\hline \multicolumn{3}{|l|}{ Plasmids } \\
\hline pBHR68 & phaCAB expression plasmid & {$[25]$} \\
\hline p68orfZ & orfZ inserted into pBHR68 & {$[24]$} \\
\hline pEn & Expression vector, $\mathrm{P}_{\text {pdc }}$ promoter & [24] \\
\hline pBBR1MCS-2 & Broad-host-range vector & {$[26]$} \\
\hline pMCSH5 & $P_{p d c}-s u c D-4 h b D$ inserted into pBBR1MCS-2 & {$[24]$} \\
\hline p68-pct-CAB & pct and phaCAB expression plasmid & {$[27]$} \\
\hline pEn-pta-ackA & pta-ackA inserted into pEn & This study \\
\hline pEn-acs & acs inserted into pEn & This study \\
\hline pEn-prpP & prpP inserted into $\mathrm{pEn}$ & This study \\
\hline pMCS-pta-ackA & $P_{\text {pdc }}-$ pta-ackA inserted into pBBR1MCS-2 & This study \\
\hline pMCS-acs & $P_{p d c}-$ acs inserted into pBBR1MCS-2 & This study \\
\hline pMCSH5-pta-ackA & $P_{p d c}-p t a-a c k A$ inserted into pMCSH5 & This study \\
\hline pMCS-pta-ackA-prpP & $P_{p d c}-p t a-a c k A-P_{p d c}-p r p P$ inserted into pBBR1MCS-2 & This study \\
\hline
\end{tabular}

medium (MM). TB medium contained $24 \mathrm{~g} / \mathrm{L}$ yeast extract, $12 \mathrm{~g} / \mathrm{L}$ Bacto tryptone, $2.31 \mathrm{~g} / \mathrm{L} \mathrm{KH}_{2} \mathrm{PO}_{4}$, and $12.54 \mathrm{~g} / \mathrm{L} \mathrm{K}_{2} \mathrm{HPO}_{4} . \mathrm{MM}$ medium comprised $2 \mathrm{~g} / \mathrm{L} \mathrm{NH}_{4} \mathrm{Cl}$, $5.0 \mathrm{~g} / \mathrm{L}\left(\mathrm{NH}_{4}\right)_{2} \mathrm{SO}_{4}, 6.0 \mathrm{~g} / \mathrm{L} \quad \mathrm{KH}_{2} \mathrm{PO}_{4}, 8.4 \mathrm{~g} / \mathrm{L}$ MOPS, $0.5 \mathrm{~g} / \mathrm{L} \mathrm{NaCl}, 0.24 \mathrm{~g} / \mathrm{L} \mathrm{MgSO}_{4}, 0.002 \mathrm{~g} / \mathrm{L} \mathrm{Na}_{2} \mathrm{MoO}_{4}$ and $1 \mathrm{~mL} / \mathrm{L}$ trace element solution. The trace elements solution contained $(\mathrm{g} / \mathrm{L}): \mathrm{FeCl}_{2} \cdot 4 \mathrm{H}_{2} \mathrm{O} 3.6, \mathrm{CaCl}_{2} \cdot 2 \mathrm{H}_{2} \mathrm{O}$ 5.0, $\mathrm{MnCl}_{2} \cdot 2 \mathrm{H}_{2} \mathrm{O} \quad 1.3, \mathrm{CuCl}_{2} \cdot 2 \mathrm{H}_{2} \mathrm{O} \quad 0.38, \mathrm{CoCl}_{2} \cdot 6 \mathrm{H}_{2} \mathrm{O} \quad 0.5$, $\mathrm{ZnCl}_{2}$ 0.94, $\mathrm{H}_{3} \mathrm{BO}_{3} 0.0311, \mathrm{Na}_{2}$ EDTA. $2 \mathrm{H}_{2} \mathrm{O} 0.4$, and thiamine- $\mathrm{HCl} 1.01$. When necessary, MM medium was supplemented with a certain amount of yeast extract.

Plasmids pMCSH5 and pMCSH5-pta-ackA, each together with phaCAB-orfZ expression plasmid p68orfZ, were co-transformed into $E$. coli JM109SG to generate $\mathrm{P} 3 \mathrm{HB} 4 \mathrm{HB}$ producing strains. The resulting recombinants were cultivated in MM medium supplemented with $10 \mathrm{~g} / \mathrm{L}$ yeast extract and $5 \mathrm{~g} / \mathrm{L}$ acetate for P3HB4HB accumulation. When necessary, $\alpha$-ketoglutarate or citrate was added as a second carbon source at a concentration of $1 \mathrm{~g} / \mathrm{L}$.

Plasmids pBBR1MCS-2, pMCS-pta-ackA, and pMCS-pta-ackA-prpP, each together with pct-phaCAB expression plasmid p68-pct-CAB, were co-transformed into E. coli JM109 to generate PHBV producing strains. The resulting recombinants were cultivated in $\mathrm{MM}$ medium supplemented with $10 \mathrm{~g} / \mathrm{L}$ yeast extract, $5 \mathrm{~g} / \mathrm{L}$ acetate, and $1.5 \mathrm{~g} / \mathrm{L}$ propionate for PHBV production.

\section{Analytical methods}

Bacterial cells were harvested by centrifugation at $8000 \mathrm{~g}$ for $10 \mathrm{~min}$. The cell pellets were washed twice with distilled water and then lyophilized for $12 \mathrm{~h}$ for cell dry weight (CDW) measurement. Lyophilized cells were subjected to methanolysis at $100{ }^{\circ} \mathrm{C}$ for $4 \mathrm{~h}$ in the presence of $3 \%(\mathrm{v} / \mathrm{v}) \mathrm{H}_{2} \mathrm{SO}_{4}$. Intracellular polymer content (wt\%) and composition was analyzed using gas chromatography (GC) (Hewlett-Packard 6890) equipped with a capillary column HP-5 (30 m, $0.25 \mathrm{~mm}$ ) and a flame-ionization detector. PHBV and $\gamma$-butyrolactone purchased from Sigma-Aldrich (St. Louis, MO, USA) were used as analysis standards. Residual cell weight (rCDW) is defined as non-PHA cell mass. rCDW in each sample was determined by substracting the PHA concentration measured by GC analysis from CDW. 
For acetate detection, the supernatant of culture broth was filtered through a $0.2-\mu \mathrm{m}$ syringe filter and stored chilled for high-performance liquid chromatography (HPLC) analysis equipped with an ion exchange column (Aminex ${ }^{\circledR} \mathrm{HPX}-87 \mathrm{H}$, BioRad) and a refractive index detector (RI-150, Thermo Spectra System, USA). A mobile phase of $7 \mathrm{mM} \mathrm{H}_{2} \mathrm{SO}_{4}$ at a $0.6 \mathrm{~mL} / \mathrm{min}$ flow rate was used.

\section{Results and discussion}

\section{Pathway construction for P3HB synthesis from acetate}

Over the past few decades, researchers have mainly been studying the "acetate switch" with the aim to enable microorganisms to efficiently consume acetate that is produced by their own metabolism to eliminate the toxicity of acetate to cell growth $[28,29]$. Nonetheless, acetate could be a potential cost-effective feedstock for synthesis of value-added chemicals. Recently, the feasibility of converting acetate to poly-3-hydroxybutyrate (P3HB) and succinate was demonstrated in Y. lipolytica [30] and E. coli [23], respectively. Moreover, co-production of hydrogen and $\mathrm{P} 3 \mathrm{HB}$ with engineered $E$. coli on glucose and acetate under anaerobic condition was reported. The addition of acetate to the culture as part of carbon source significantly increased $\mathrm{P} 3 \mathrm{HB}$ production, yet the $\mathrm{P} 3 \mathrm{HB}$ titer was still below $0.04 \mathrm{~g} / \mathrm{L}$ [31].

To investigate the possibility of $\mathrm{P} 3 \mathrm{HB}$ production using acetate as a main carbon source in $E$. coli, P3HB synthesis operon phaCAB carrying plasmid pBHR68 was transformed into E. coli JM109, and the recombinant strain was inoculated into MM medium supplemented with $2 \mathrm{~g} / \mathrm{L}$ yeast extract and $5 \mathrm{~g} / \mathrm{L}$ acetate (Fig. 2). After $48 \mathrm{~h}$ shake flask cultivation, E. coli JM109 (pBHR68) consumed all the acetate and yielded $1.49 \mathrm{~g} / \mathrm{L}$ CDW with $0.22 \mathrm{~g} / \mathrm{L}$ P3HB accumulation, which indicated that P3HB could be produced from acetate, yet the P3HB titer was much lower than that obtained from glucose [32]. Therefore, further metabolic engineering strategies were applied to improve $\mathrm{P} 3 \mathrm{HB}$ accumulation from acetate.

\section{Pathway engineering for improved P3HB accumulation}

In $E$. coli, there are two potential routes for the generation of acetyl-CoA from acetate: phosphotransacetylase/ acetate kinase (pta-ackA pathway) and AMP-forming acetyl-CoA synthetase (acs pathway) (Fig. 1). These two pathways were augmented through the overexpression of $p t a-a c k A$ and acs genes, respectively. Shake flask cultivations in acetate containing MM medium showed that
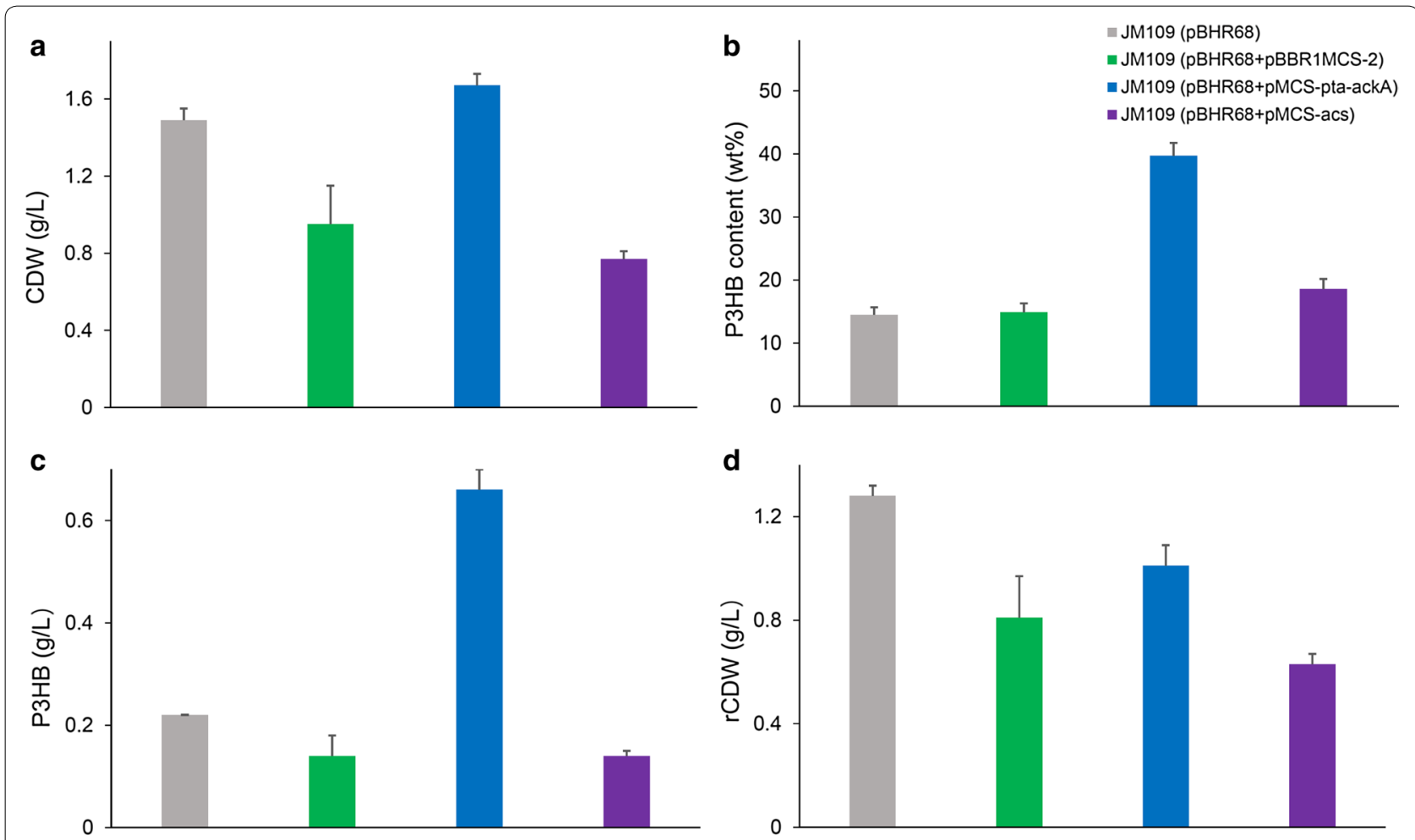

Fig. 2 Effects of the overexpression of pta-ackA and acs on cell growth and P3HB production. E. coli recombinants harboring different plasmids were cultivated in MM medium supplemented with $5 \mathrm{~g} / \mathrm{L}$ acetate and $2 \mathrm{~g} / \mathrm{L}$ yeast extract at $37^{\circ} \mathrm{C}$ for $48 \mathrm{~h}$. CDW (a), P3HB content (b), P3HB titer (c), and $\mathrm{rCDW}(\mathbf{d})$ were measured. The columns represent the averages of triplicate experiments, and the error bars represent standard deviation 
JM109 (pBHR68+pMCS-pta-ackA) consumed $5 \mathrm{~g} / \mathrm{L}$ acetate and produced $0.66 \mathrm{~g} / \mathrm{L}$ P3HB. The $\mathrm{P} 3 \mathrm{HB}$ production titer of was roughly four times of that produced by control strain JM109 (pBHR68 + pBBR1MCS-2). By contrast, the overexpression of acs gene has little effect on improving $\mathrm{P} 3 \mathrm{HB}$ accumulation (Fig. 2). The activation of each molecule of acetate for acetyl-CoA synthesis in acs pathway requires two molecule of ATP, while pta-ackA pathway consumes only one molecule of ATP for acetylCoA synthesis $[22,33]$. It was reported also that the growth of $E$. coli stains on low concentrations of acetate depends on acs pathway, while growth on high concentrations requires $p t a-a c k A$ pathway $[28,29]$. Therefore, the engineering of the pta-ackA pathway to strengthen acetate assimilation was considered to be an effective strategy for improving $\mathrm{P} 3 \mathrm{HB}$ production in recombinant E. coli.

\section{Optimizing culture conditions to increase $\mathrm{P} 3 \mathrm{HB}$ production} The engineered E. coli JM109 (pBHR68+pMCSpta-ackA) and control strain JM109 (pBHR68 + pBBR1MCS-2) were cultivated in shake flasks with $\mathrm{LB}, \mathrm{TB}$, and MM medium to evaluate the effects of medium composition on cell growth and $\mathrm{P} 3 \mathrm{HB}$ accumulation (Fig. 3). Initial acetate concentration was $5 \mathrm{~g} / \mathrm{L}$ and $\mathrm{MM}$ medium was supplemented with $2 \mathrm{~g} / \mathrm{L}$ yeast extract.
After $48 \mathrm{~h}$ cultivation, no acetate was left in the medium for all groups. The recombinant strain harboring extra pta-ackA genes produced more CDW and $\mathrm{P} 3 \mathrm{HB}$ in the three different kinds of culture media, further demonstrated that pta-ackA overexpression was effective for improving P3HB production from acetate (Fig. 3). With MM medium, JM109 (pBHR68 + pMCS-pta-ackA) produced $1.67 \mathrm{~g} / \mathrm{L} \mathrm{CDW}$, containing $0.66 \mathrm{~g} / \mathrm{L} \mathrm{P} 3 \mathrm{HB}$, while the use of $\mathrm{LB}$ and TB medium resulted in $1.55 \mathrm{~g} / \mathrm{L}$ CDW with $0.52 \mathrm{~g} / \mathrm{L} \mathrm{P} 3 \mathrm{HB}$, and $1.93 \mathrm{~g} / \mathrm{L} \mathrm{CDW}$ with $0.74 \mathrm{~g} / \mathrm{L}$ P3HB, respectively. TB medium helped to obtain higher CDW and $\mathrm{P} 3 \mathrm{HB}$ titer than those achieved in $\mathrm{MM}$ and $\mathrm{LB}$ medium. Among three culture conditions, MM medium possesses lowest cost and resulted in acceptable $\mathrm{P} 3 \mathrm{HB}$ titer. Therefore, it was considered to be a favorable medium for the production of $\mathrm{P} 3 \mathrm{HB}$ from acetate.

Next, different concentration $(0,2,4,6,8,10 \mathrm{~g} / \mathrm{L})$ of yeast extract were added to $\mathrm{MM}$ medium to study its potential for enhancing cell growth and $\mathrm{P} 3 \mathrm{HB}$ accumulation (Fig. 4). When yeast extract was not provided, CDW of E. coli JM109 (pBHR68+ pMCS-pta-ackA) reached $1.03 \mathrm{~g} / \mathrm{L}$, and $\mathrm{P} 3 \mathrm{HB}$ concentration was only $0.02 \mathrm{~g} / \mathrm{L}$. The CDW and P3HB accumulation increased gradually with the increase of yeast extract concentration, although the intracellular P3HB content did not change obviously. The addition of $10 \mathrm{~g} / \mathrm{L}$ yeast extract yielded $3.02 \mathrm{~g} / \mathrm{L} \mathrm{CDW}$,
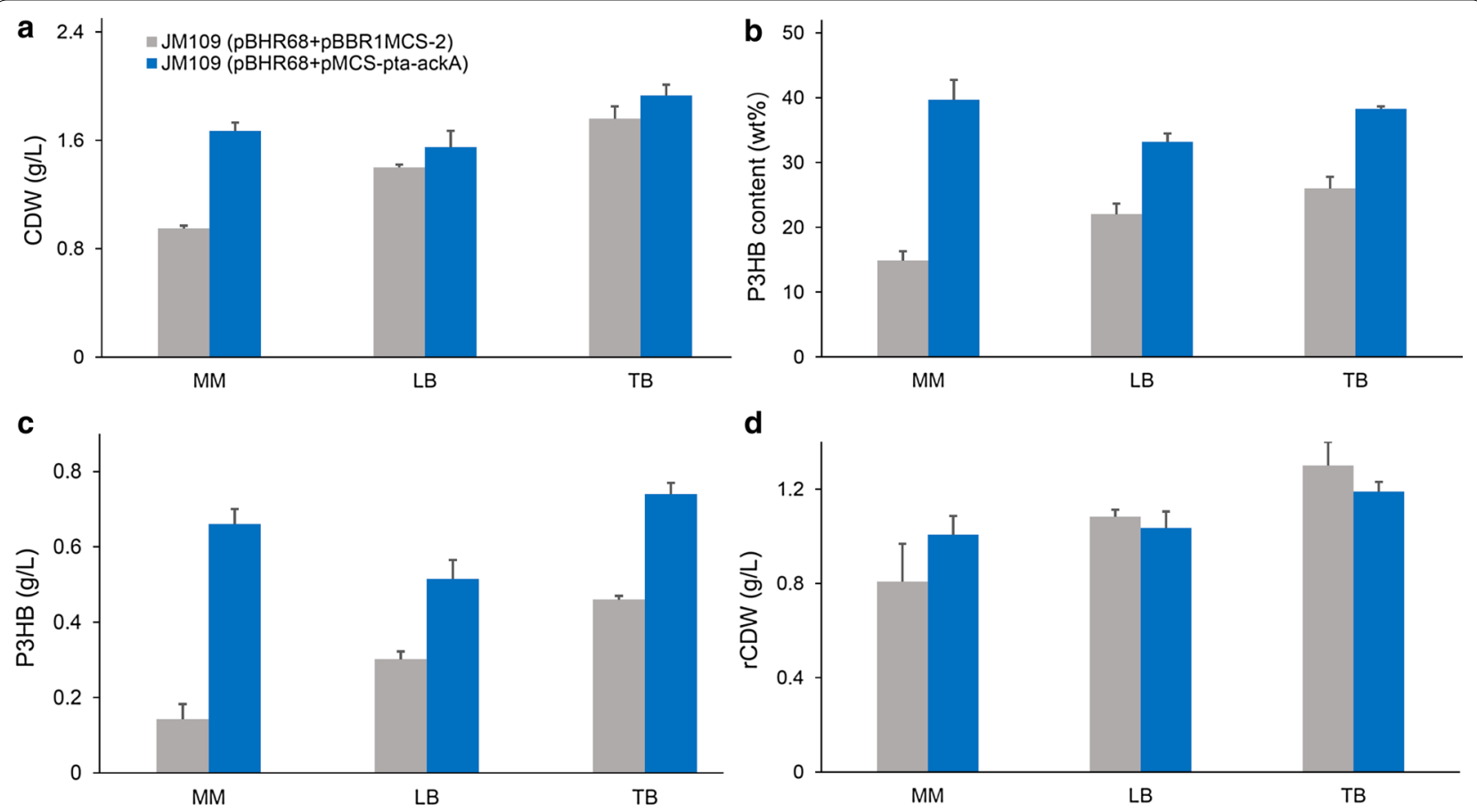

Fig. 3 Effects of medium composition on cell growth and P3HB production. E. coli recombinants harboring different plasmids were cultivated in $\mathrm{MM}$, LB, or TB medium supplemented with $5 \mathrm{~g} / \mathrm{L}$ acetate at $37^{\circ} \mathrm{C}$ for $48 \mathrm{~h}$. MM medium contained $2 \mathrm{~g} / \mathrm{L}$ yeast extract. CDW (a), P3HB content (b), P3HB titer (c), and rCDW (d) were measured. The columns represent the averages of triplicate experiments, and the error bars represent standard deviation 

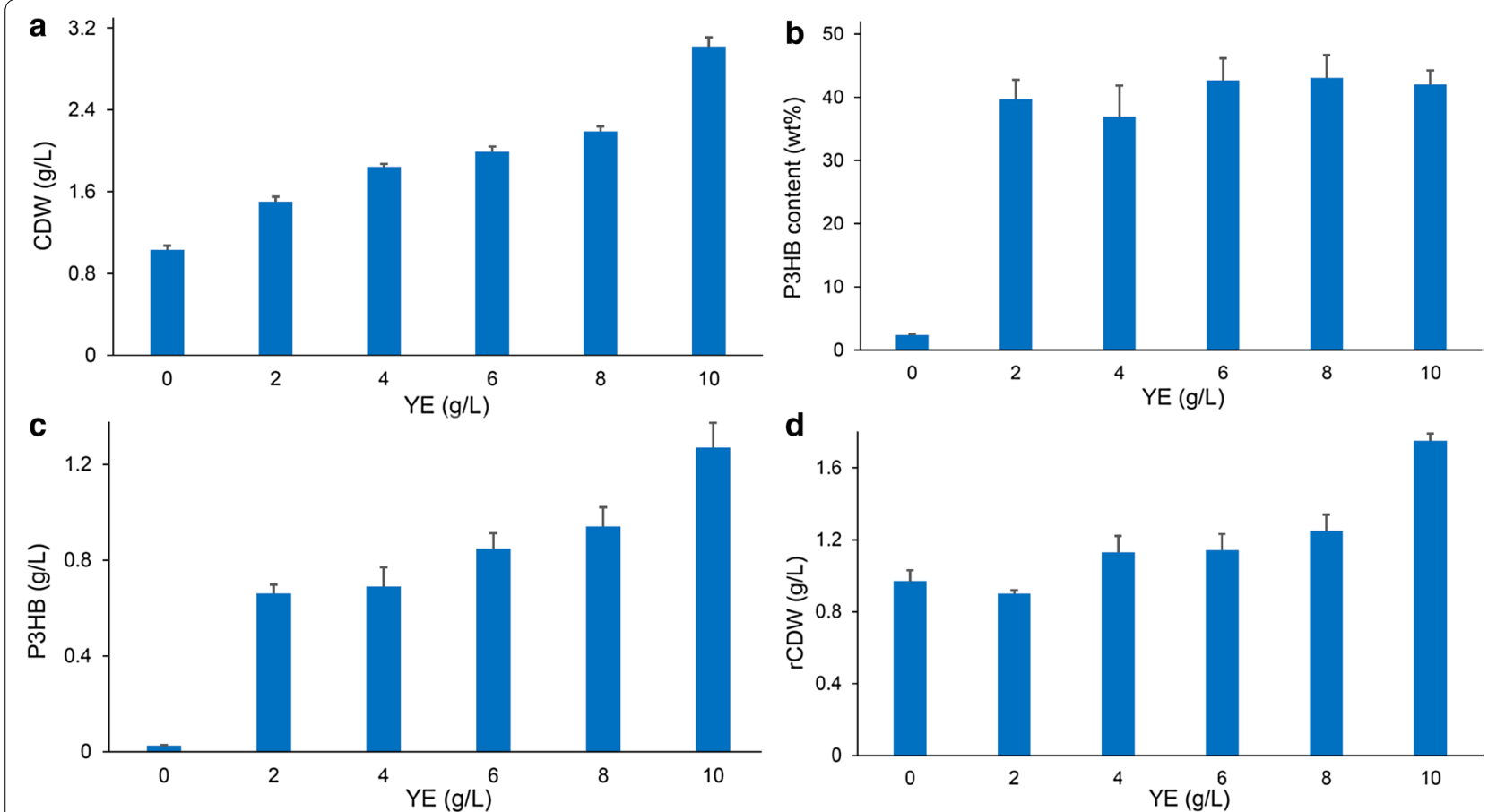

Fig. 4 Effect of yeast extract concentration on cell growth and P3HB production. E. coli JM109 (pBHR68 + pMCS-pta-ackA) was cultivated in MM medium supplemented with $5 \mathrm{~g} / \mathrm{L}$ acetate at $37^{\circ} \mathrm{C}$ for $48 \mathrm{~h}$. Different concentration $(0,2,4,6,8,10 \mathrm{~g} / \mathrm{L}$ ) of yeast extract (YE) was added to the culture. CDW (a), P3HB content (b), P3HB titer (c), and rCDW (d) were measured. The columns represent the averages of triplicate experiments, and the error bars represent standard deviation

containing $1.27 \mathrm{~g} / \mathrm{L}$ P3HB, which was the highest P3HB production titer obtained in this study. The production yield was $0.25 \mathrm{~g} \mathrm{P} 3 \mathrm{HB} / \mathrm{g}$ acetate, which was $35 \%$ of the maximal theoretical yield. It has been shown that yeast extract contributes a large amount of amino acids for biomass growth, thus the addition of complex nitrogen sources such as yeast extract to defined medium saved NADPH for amino acids synthesis and increased intracellular NADPH level as well as NADPH/NADP ratio [34]. P3HB biosynthesis is a NADPH-dependent pathway and high NADPH level and availability were considered to be of great importance for the efficient synthesis of PHB in recombinant E. coli [32]. Therefore, we speculate that the addition of yeast extract favored cell growth and intracellular NADPH availability which in turn led to high biomass and $\mathrm{P} 3 \mathrm{HB}$ production.

We next cultivated E. coli JM109 (pBHR68 + pMCSpta-ackA) using MM medium supplemented with $10 \mathrm{~g} / \mathrm{L}$ yeast extract and different concentration $(0,1,2,3,4$, $5 \mathrm{~g} / \mathrm{L}$ ) of acetate to evaluate the effect of acetate concentration on $\mathrm{P} 3 \mathrm{HB}$ accumulation. As shown in Additional file 1: Figure S1, when acetate was not provided, the CDW reached $1.15 \mathrm{~g} / \mathrm{L}$ and the intracellular P3HB content was only $3.23 \mathrm{wt} \%$. With the increase of acetate concentration, the CDW, $\mathrm{P} 3 \mathrm{HB}$ content, and $\mathrm{P} 3 \mathrm{HB}$ titer all increased gradually. The addition of $5 \mathrm{~g} / \mathrm{L}$ acetate led to the highest $\mathrm{P} 3 \mathrm{HB}$ content of $42.02 \mathrm{wt} \%$. Although yeast extract was provided at a relatively high level, $\mathrm{P} 3 \mathrm{HB}$ synthesis was still limited by acetate addition. In contrast, the results of shake flask cultures using $5 \mathrm{~g} / \mathrm{L}$ acetate and different amounts of yeast extract showed that the P3HB content did not change obviously when yeast extract concentration increased from 2 to $10 \mathrm{~g} / \mathrm{L}$ (Fig. 4). These results demonstrated that the carbon source existed in yeast extract was not able to support effective P3HB synthesis, and acetate was the main carbon source to synthesize P3HB.

\section{Production of P3HB4HB from acetate}

Poly(3-hydroxybutyrate-co-4-hydroxybutyrate) (P3HB4HB) exhibits favorable biodegradability and a wide range of physical properties ranging from highly crystalline plastic to elastic rubber, thus it is considered to be one of the most promising PHA materials. The synthesis of P3HB4HB copolymer from structural unrelated carbon sources such as glucose in E. coli has been reported $[24,35,36]$. Nevertheless, in terms of substrate cost, acetate is a promising carbon source for microbial fermentations. Therefore, we aimed to developed an 
engineered $E$. coli that can produce $\mathrm{P} 3 \mathrm{HB} 4 \mathrm{HB}$ from acetate as carbon source.

The genes involved in succinate degradation pathway of Clostridium kluyveri, including sucD, $4 \mathrm{hbD}$, and $\operatorname{orf} Z$ [37], was combined with $\mathrm{P} 3 \mathrm{HB}$ synthesis operon phaCAB to construct $\mathrm{P} 3 \mathrm{HB} 4 \mathrm{HB}$ producing pathway. E. coli native succinate semialdehyde dehydrogenase genes sad and gabD were both deleted for eliminating succinate formation from succinate semialdehyde (Fig. 1). The resulting strain JM109SG (p68orfZ + pMCSH5) was able to achieve $2.37 \mathrm{~g} / \mathrm{L}$ CDW containing 35.79 wt\% $\mathrm{P}(3 \mathrm{HB}-\mathrm{co}-9.48 \mathrm{~mol} \%$ $4 \mathrm{HB})$ when grown in mineral medium supplemented with $10 \mathrm{~g} / \mathrm{L}$ yeast extract and $5 \mathrm{~g} / \mathrm{L}$ acetate (Fig. 5). In addition, the $p t a-a c k A$ genes were overexpressed to strengthen acetate assimilation, and the recombinant JM109SG (p68orfZ + pMCSH5-pta-ackA) yielded $2.96 \mathrm{~g} / \mathrm{L} \mathrm{CDW}$, containing $58.01 \mathrm{wt} \% \mathrm{P}(3 \mathrm{HB}-$ co-5.79 mol\% 4HB) (Fig. 5). In terms of P3HB4HB production titer, pta-ackA overexpression resulted in $1.71 \mathrm{~g} / \mathrm{L}$, significantly higher than $0.85 \mathrm{~g} / \mathrm{L}$ of the control strain without pta-ackA overexpression. These results were consistent with previous $\mathrm{P} 3 \mathrm{HB}$ producing studies, and it appears that the pta-ackA plasmid carrying recombinants were more productive than the strains without $p t a-a c k A$ overexpression.

\section{Influence of TCA cycle intermediates on P3HB4HB synthesis}

Previously, the addition of TCA cycle intermediates including $\alpha$-ketoglutarate and citrate were found to be effective for increasing metabolic flux into $4 \mathrm{HB}$ precursor when glucose was employed as carbon source [24]. Therefore, shake flask cultures were performed to evaluate the effects of $\alpha$-ketoglutarate and citrate addition on cell growth and P3HB4HB accumulation profiles in case of acetate as a main carbon source. As shown in Fig. 5, when $1 \mathrm{~g} / \mathrm{L}$ of $\alpha$-ketoglutarate was added, the recombinant $E$. coli reached $3.63 \mathrm{~g} / \mathrm{L} C D W$, containing $54.97 \mathrm{wt} \%$ $\mathrm{P}(3 \mathrm{HB}-$ co-5.80 mol\% 4HB). Similarly, the addition of $1 \mathrm{~g} / \mathrm{L}$ citrate as joint carbon source resulted in $3.62 \mathrm{~g} / \mathrm{L}$ CDW, containing $59.48 \mathrm{wt} \% \mathrm{P}(3 \mathrm{HB}-c o-3.43 \mathrm{~mol} \%$ 4HB). The addition of $\alpha$-ketoglutarate and citrate as additional carbon sources increased the cell growth and P3HB4HB production, but had no obvious effect on improving $4 \mathrm{HB}$ monomer content.

When $E$. coli metabolizes acetate as a sole carbon and energy source, the genes involved in acetate uptake, glyoxylate cycle, TCA cycle, and gluconeogenesis were all up-regulated [12]. The addition of $\alpha$-ketoglutarate or citrate could improve the supply of TCA cycle intermediates, thus may benefit cell growth and acetate assimilation. However, on this occasion, 4HB monomer content
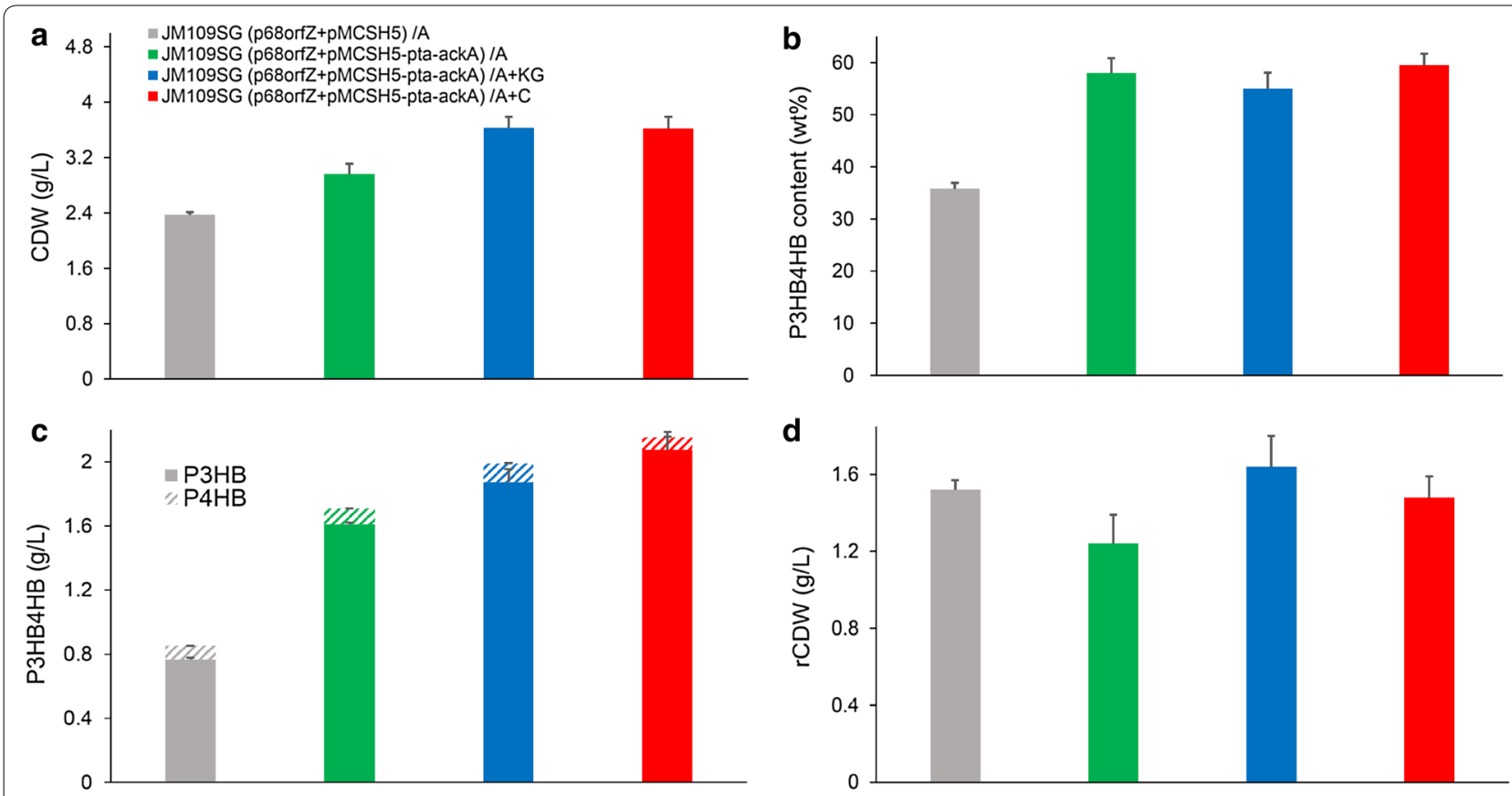

Fig. 5 P3HB4HB production by E. coli strains grown in shake flasks. E. coli recombinants harboring different plasmids were cultivated in MM medium supplemented with $10 \mathrm{~g} / \mathrm{L}$ yeast extract and different carbon sources at $37^{\circ} \mathrm{C}$ for $48 \mathrm{~h}$. Initial acetate (A) concentration was $5 \mathrm{~g} / \mathrm{L}$. a-ketoglutarate (KG) or citrate (C) was added as assistant carbon source at a concentration of $1 \mathrm{~g} / \mathrm{L}$. CDW (a), P3HB4HB content (b), P3HB4HB titer (c), and rCDW (d) were measured. The columns represent the averages of triplicate experiments, and the error bars represent standard deviation 
was not increased, indicating the metabolic flux towards $4 \mathrm{HB}$ precursor synthesis was still limited in spite of improved TCA cycle intermediate supply.

\section{Production of PHBV from acetate and propionate}

Poly(3-hydroxybutyrate-co-3-hydroxyvalerate) (PHBV) is more flexible and tougher than $\mathrm{P} 3 \mathrm{HB}$ homopolymer and has been commercially produced for many years [7]. The normal PHBV synthesis process requires the addition of propionate as assistant carbon source to generate propionyl-CoA, which was the precursor of 3-hydroxyvalerate (3HV) monomer (Fig. 1) [38]. The conversion of propionate to propionyl-CoA could be catalyzed by the ATPdependent propionyl-CoA synthetase or propionyl-CoA transferase (Pct). When acetate was employed as carbon source, the intracellular pool size of acetyl-CoA should be favorable for transferring a CoA group from acetylCoA to propionate. To study the possibility of PHBV synthesis from acetate and propionate, plasmid p68pct-CAB, harboring Megasphaera elsdenii propionylCoA transferase and $\mathrm{P} 3 \mathrm{HB}$ synthesis operon phaCAB, was co-transformed with pMCS-pta-ackA into $E$. coli JM109. The resulting recombinant reached $2.64 \mathrm{~g} / \mathrm{L}$ CDW, containing $12.52 \mathrm{wt} \% \mathrm{P}(3 \mathrm{HB}-\mathrm{co}-6.58 \mathrm{~mol} \%$ 3HV) (Fig. 6). In contrast, the control strain JM109 (p68-pct$\mathrm{CAB}+\mathrm{pBBR} 1 \mathrm{MCS}-2)$ only reached $2.36 \mathrm{~g} / \mathrm{L} \mathrm{CDW}$ containing $6.48 \mathrm{wt} \% \mathrm{P}(3 \mathrm{HB}-\mathrm{co}-15.80 \mathrm{~mol} \% 3 \mathrm{HV})$ under the same culture condition. PHBV production titer was increased from 0.15 to $0.33 \mathrm{~g} / \mathrm{L}$ with $p t a-a c k A$ overexpression, further demonstrating that pta-ackA overexpression was an effective strategy for improving acetate-dependent biopolymer synthesis.

Previously, recombinant E. coli harboring phaCAB operon and propionate permease (or propionyl-CoA synthase) failed to incorporate $3 \mathrm{HV}$ into the biopolymer in M9 medium containing $2 \mathrm{~g} / \mathrm{L}$ yeast extract, $1 \mathrm{~g} / \mathrm{L}$ sodium propionate, and $20 \mathrm{~g} / \mathrm{L}$ sodium acetate [39]. However, a high concentration of yeast extract $(10 \mathrm{~g} / \mathrm{L})$ was used in this study. It was speculated that a high concentration of yeast extract favors cell growth. In addition, propionyl-CoA transferase used acetyl-CoA and propionate to produce propionyl-CoA, yielding the precursor for $3 \mathrm{HV}$ incorporation. Therefore, our engineering strategy suggest that PHBV could be produced from acetate and propionate, yet the biopolymer content was lower than that achieved for $\mathrm{P} 3 \mathrm{HB}$ or $\mathrm{P} 3 \mathrm{HB} 4 \mathrm{HB}$.

Next, prpP gene encoding propionate permease of $R$. eutropha $\mathrm{H} 16$ was overexpressed to study its effect on PHBV synthesis. E. coli JM109 (p68-pct-CAB + pMCSpta-ackA-prpP) resulted in $3.15 \mathrm{~g} / \mathrm{L} \mathrm{CDW}$, containing $34.77 \mathrm{wt} \% \mathrm{P}(3 \mathrm{HB}-\mathrm{co}-10.37 \mathrm{~mol} \%$ 3HV) (Fig. 6). The overexpression of propionate permease improved
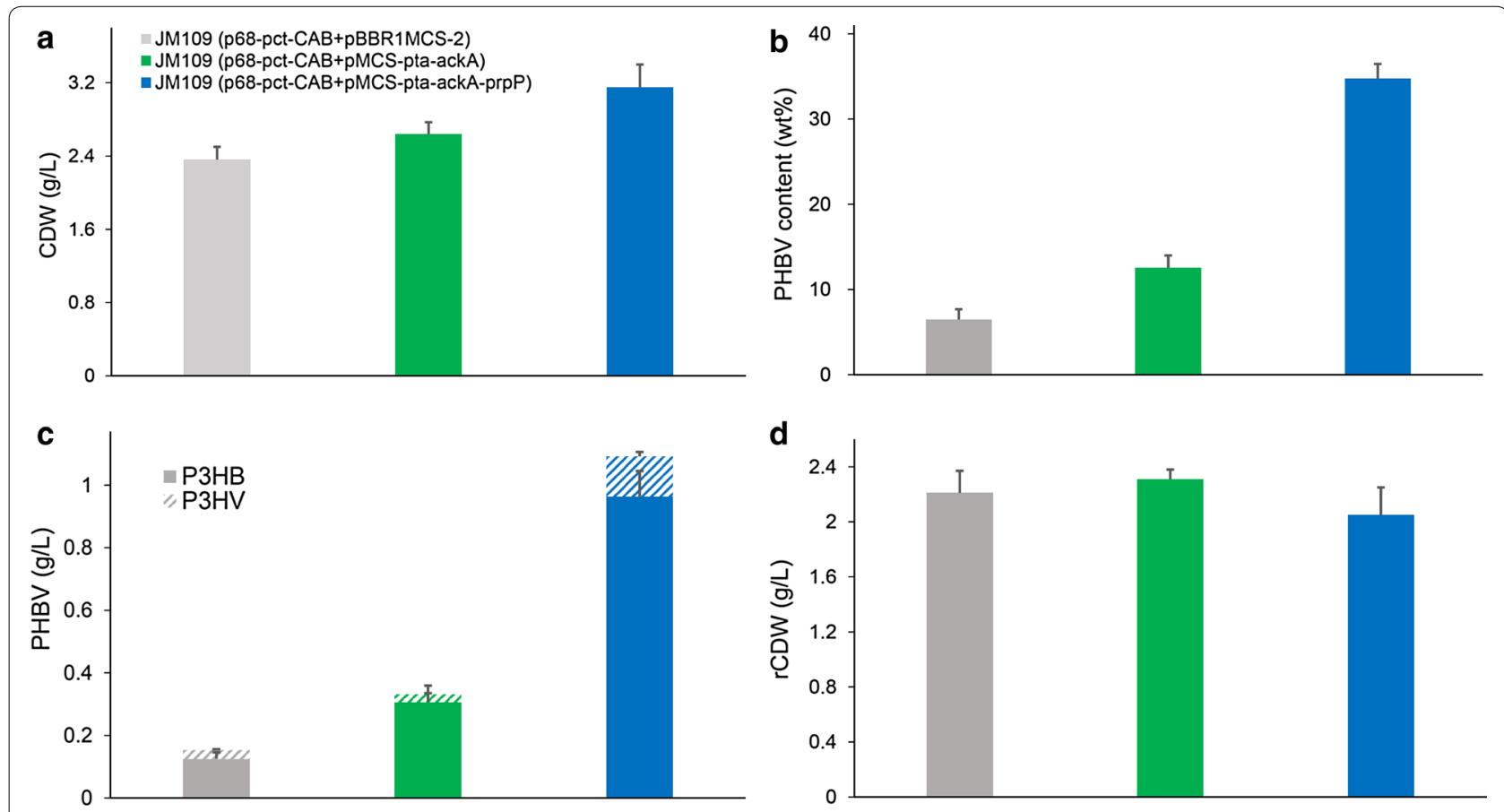

Fig. 6 PHBV production by E. coli strains grown in shake flasks. E. coli recombinants harboring different plasmids were cultivated in MM medium supplemented with $10 \mathrm{~g} / \mathrm{L}$ yeast extract, $5 \mathrm{~g} / \mathrm{L}$ acetate, and $1.5 \mathrm{~g} / \mathrm{L}$ propionate at $37^{\circ} \mathrm{C}$ for $48 \mathrm{~h}$. CDW (a), PHBV content (b), PHBV titer (c), and rCDW (d) were measured. The columns represent the averages of triplicate experiments, and the error bars represent standard deviation 
PHBV production titer from 0.33 to $1.09 \mathrm{~g} / \mathrm{L}$. Meanwhile, 3HV monomer content was increased from 6.58 to $10.37 \mathrm{~mol} \%$. Similar phenomenon were also observed in previous studies, in which overexpression of PrpP not only increased the $3 \mathrm{HV}$ monomer content but also promoted the biopolymer accumulation [40]. Propionate permease is responsible for the uptake of propionate and PrpP overexpression may improve the pool of intracellular propionate for propionyl-CoA formation. Thus, it was reasonable to find that $3 \mathrm{HV}$ fraction in PHBV was increased with PrpP overexpression. However, the reason for improved biopolymer production caused by PrpP overexpression was not clear and need to be discussed further.

Use of corn steep liquor as an economical nitrogen source Yeast extract was proved to be a critical nutritional supplement for improved PHA production (Fig. 4). To reduce the feedstock cost, many studies tried to develop cheaper supplements which can be used as an alternative or in combination with yeast extract. Corn steep liquor, a low-cost byproduct of the corn wet-milling process, contains a large amount of amino acids, peptides and vitamins [41]. Therefore, there have been increasing studies on the utilization of corn steep liquor as a nitrogen source for microbial fermentations [42, 43].

Shake flask cultivations using various concentration (2, $4,6,8,10 \mathrm{~g} / \mathrm{L}$ ) of corn steep liquor instead of yeast extract were performed (Fig. 7). The CDW of E. coli JM109 (pBHR68 + pMCS-pta-ackA) increased gradually with the increase of corn steep liquor concentration, yet the P3HB production exhibit a trend of first increasing then decreasing. The addition of $4 \mathrm{~g} / \mathrm{L}$ and $6 \mathrm{~g} / \mathrm{L}$ of corn steep liquor to $\mathrm{MM}$ medium containing $5 \mathrm{~g} / \mathrm{L}$ acetate yielded 0.91 and $0.96 \mathrm{~g} / \mathrm{L} \mathrm{P3HB}$, respectively. Although $10 \mathrm{~g} / \mathrm{L}$ corn steep liquor addition led to highest CDW among the different corn steep liquor concentration condition, the $\mathrm{P} 3 \mathrm{HB}$ concentration was only $0.56 \mathrm{~g} / \mathrm{L}$. Therefore, the favorable corn steep liquor concentration for $\mathrm{P} 3 \mathrm{HB}$ production was 4-6 g/L under our culture condition. These results indicated that $\mathrm{P} 3 \mathrm{HB}$ can be synthesized when corn steep liquor is employed as a nitrogen source, albeit with somewhat lower efficiency in comparison with yeast extract. The use of corn steep liquor for PHA production from acetate therefore merits further research.

In addition, we performed shake flask studies without acetate addition to evaluate the contribution of CSL to P3HB production. As shown in Additional file 1: Table S2,

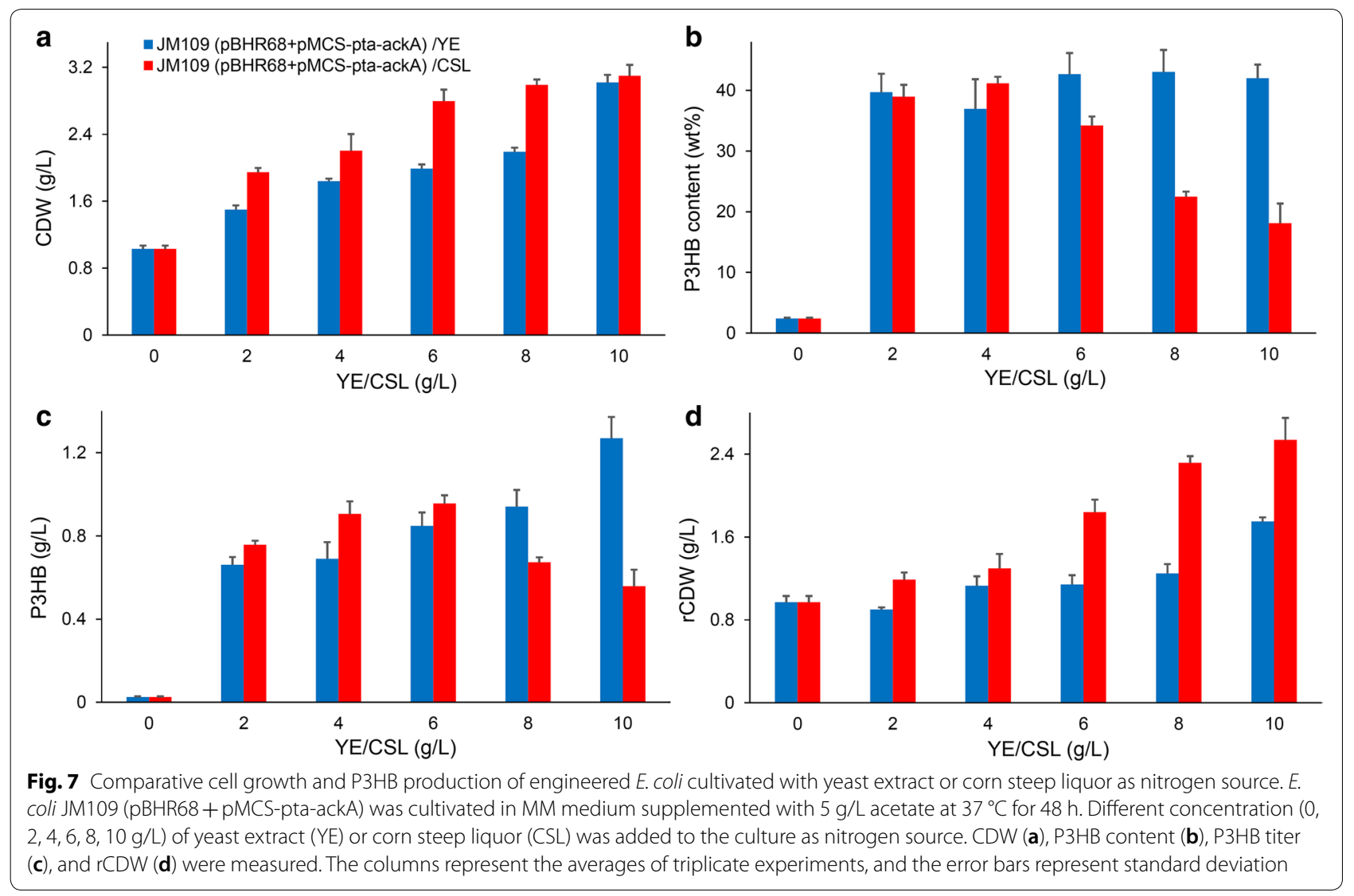


E. coli JM109 (pBHR68+ pMCS-pta-ackA) showed the highest $\mathrm{P} 3 \mathrm{HB}$ accumulation of $0.04 \mathrm{~g} / \mathrm{L}$ among all strains studied when cultivated in MM medium supplemented with $6 \mathrm{~g} / \mathrm{L}$ CSL. The P3HB titer obtained without acetate was much lower than that achieved by acetate supplementation. These results further demonstrated that acetate was served as the main carbon source to support effective P3HB synthesis.

\section{Conclusions}

In this study, E. coli was engineered to synthesize P3HB, P3HB4HB, and PHBV using acetate as a main carbon source. The production of biopolymers was significantly improved by the overexpression of phosphotransacetylase and acetate kinase. In shake flask cultures, the engineered E. coli produced $1.27 \mathrm{~g} / \mathrm{L}$ of P3HB and $1.71 \mathrm{~g} / \mathrm{L}$ $\mathrm{P}(3 \mathrm{HB}-$ co-5.79 mol\% 4HB), respectively, with minimal medium supplemented $10 \mathrm{~g} / \mathrm{L}$ yeast extract and $5 \mathrm{~g} / \mathrm{L}$ acetate. When $1 \mathrm{~g} / \mathrm{L}$ citrate was added as assistant carbon source, P3HB4HB production titer was increased to $2.15 \mathrm{~g} / \mathrm{L}$. The overexpression of propionyl-CoA transferase and propionate permease lead to a production titer of $1.09 \mathrm{~g} / \mathrm{L} \mathrm{P}(3 \mathrm{HB}-\mathrm{co}-10.37 \mathrm{~mol} \% 3 \mathrm{HV})$ when $5 \mathrm{~g} / \mathrm{L}$ acetate and $1.5 \mathrm{~g} / \mathrm{L}$ propionate were simultaneously supplied. To our knowledge, this is the first study reporting the efficient production of $\mathrm{P} 3 \mathrm{HB}, \mathrm{P} 3 \mathrm{HB} 4 \mathrm{HB}$, and PHBV using acetate as a main carbon source by engineered $E$. coli.

\section{Additional file}

Additional file 1: Table S1. Oligonucleotides used in this study. Table S2. $\mathrm{P} 3 \mathrm{HB}$ production by E. coli strains cultivated in MM medium supplemented with CSL. Figure S1. Effect of acetate concentration on cell growth and $\mathrm{P} 3 \mathrm{HB}$ production.

\section{Abbreviations}

PHA: polyhydroxyalkanoates; P3HB: poly-3-hydroxybutyrate; P3HB4HB: poly(3hydroxybutyrate-co-4-hydroxybutyrate); $\mathrm{PHBV}$ : poly(3-hydroxybutyrate-co3-hydroxyvalerate); 3HB: 3-hydroxybutyrate; $4 \mathrm{HB}$ : 4-hydroxybutyrate; $3 \mathrm{HV}$ : 3-hydroxyvalerate; LB: Luria-Bertani; TB: terrific broth; MM: minimal medium; YE: yeast extract; CSL: corn steep liquor; A: acetate; KG: a-ketoglutarate; C: citrate; CDW: cell dry weight; rCDW: residual cell dry weight; GC: gas chromatography; HPLC: high-performance liquid chromatography.

\section{Authors' contributions}

$J C$ and WL performed the experiments; ZZZ assisted the gas chromatography analysis; ZJL wrote the manuscript; ZJL and TWT supervised this study. All authors read and approved the final manuscript.

\section{Author details}

${ }^{1}$ Beijing Advanced Innovation Center for Soft Matter Science and Engineering, Beijing University of Chemical Technology, Mailbox 53, No. 15 Beisanhuan Donglu, Chaoyang District, Beijing 100029, China. ${ }^{2}$ Beijing Key Laboratory of Bioprocess, College of Life Science and Technology, Beijing University of Chemical Technology, Beijing 100029, China.
Acknowledgements

Not applicable.

\section{Competing interests}

The authors declare that they have no competing interests.

Availability of data and materials

All data generated during this study are included in this published article.

Consent for publication

All authors agree to submit the work to the journal.

Ethics approval and consent to participate

Not applicable.

Funding

This research was supported by Grants from National Natural Science Foundation of China (21476014), National Science \& Technology Pillar Program of China (2015BAD15B09), Higher Education and Highquality and Worldclass Universities (BUCTYLKXJ03) and the Fundamental Research Funds for the Central Universities (XK1701)

\section{Publisher's Note}

Springer Nature remains neutral with regard to jurisdictional claims in published maps and institutional affiliations.

Received: 25 February 2018 Accepted: 26 June 2018

Published online: 03 July 2018

\section{References}

1. Farias D, Atala DIP, Maugeri F. Improving bioethanol production by Scheffersomyces stipitis using retentostat extractive fermentation at high xylose concentration. Biochem Eng J. 2017:6:171-80.

2. Ye XT, Honda K, Morimoto Y, Okano K, Ohtake H. Direct conversion of glucose to malate by synthetic metabolic engineering. J Biotechnol. 2013;164:34-40.

3. Ma JF, Li F, Liu RM, Liang LY, Ji YL, Wei C, Jiang M, Jia HH, Ouyang P. Succinic acid production from sucrose and molasses by metabolically engineered E. coli using a cell surface display system. Biochem Eng J. 2014;91:240-9.

4. Bai FW, Anderson WA, Moo-Young M. Ethanol fermentation technologies from sugar and starch feedstocks. Biotechnol Adv. 2008;26:89-105.

5. Chen GQ, Hajnal I, Wu H, Lv L, Ye JW. Engineering biosynthesis mechanisms for diversifying polyhydroxyalkanoates. Trends Biotechnol. 2015;33:565-74.

6. Chen GQ, Jiang XR, Guo Y. Synthetic biology of microbes synthesizing polyhydroxyalkanoates (PHA). Synth Syst Biotechnol. 2016;1:236-42.

7. Chen GQ. A microbial polyhydroxyalkanoates (PHA) based bio- and materials industry. Chem Soc Rev. 2009;38:2434-46.

8. Wang Y, Yin J, Chen GQ. Polyhydroxyalkanoates, challenges and opportunities. Curr Opin Biotechnol. 2014;30:59-65.

9. Sonntag F, Kroner C, Lubuta P, Peyraud R, Horst A, Buchhaupt M, Schrader J. Engineering Methylobacterium extorquens for de novo synthesis of the sesquiterpenoid a-humulene from methanol. Metab Eng. 2015;32:82-94.

10. Christophe G, Deo JL, Kumar V, Nouaille R, Fontanille P, Larroche C. Production of oils from acetic acid by the oleaginous yeast Cryptococcus curvatus. Appl Biochem Biotechnol. 2012;167:1270-9.

11. Revelles O, Tarazona N, Garcia JL, Prieto MA. Carbon roadmap from syngas to polyhydroxyalkanoates in Rhodospirillum rubrum. Environ Microbiol. 2016:18:708-20.

12. Oh MK, Rohlin L, Kao KC, Liao JC. Global expression profiling of acetategrown Escherichia coli. J Biol Chem. 2002;277:13175-83.

13. Chi ZY, Zheng YB, Ma JW, Chen SL. Oleaginous yeast Cryptococcus curvatus culture with dark fermentation hydrogen production effluent as feedstock for microbial lipid production. Int J Hydrog Energy. 2011;36:9542-50 
14. Canganella F, Kuk SU, Morgan H, Wiegel J. Clostridium thermobutyricum: growth studies and stimulation of butyrate formation by acetate supplementation. Microbiol Res. 2002;157:149-56.

15. Quillaguamán J, Delgado O, Mattiasson B, Hatti-Kaul R. Poly( $\beta$ hydroxybutyrate) production by a moderate halophile, Halomonas boliviensis LC1. Enzyme Microb Technol. 2006;38:148-54.

16. Chen Y, Cheng JJ, Creamer KS. Inhibition of anaerobic digestion process: a review. Bioresour Technol. 2008;99:4044-64.

17. Hu P, Chakraborty S, Kumar A, Woolston B, Liu H, Emerson D, Stephanopoulos $G$. Integrated bioprocess for conversion of gaseous substrates to liquids. Proc Natl Acad Sci USA. 2016;113:3773-8.

18. Ruan ZH, Zanotti M, Wang XQ, Ducey C, Liu Y. Evaluation of lipid accumulation from lignocellulosic sugars by Mortierella isabellina for biodiesel production. Bioresour Technol. 2012;110:198-205.

19. Zhang YHP. Reviving the carbohydrate economy via multi-product lignocellulose biorefineries. J Ind Microbiol Biotechnol. 2008;35:367-75.

20. Lian JN, Garcia-Perez M, Coates R, Wu HW, Chen SL. Yeast fermentation of carboxylic acids obtained from pyrolytic aqueous phases for lipid production. Bioresour Technol. 2012;118:177-86.

21. Xu JY, Liu N, Qiao KJ, Vogg S, Stephanopoulos G. Application of metabolic controls for the maximization of lipid production in semicontinuous fermentation. Proc Natl Acad Sci USA. 2017;114:5308-16.

22. Xiao Y, Ruan ZH, Liu ZG, Wu SG, Varman AM, Liu Y, Tang YJ. Engineering Escherichia coli to convert acetic acid to free fatty acids. Biochem Eng J. 2013;76:60-9.

23. Li YJ, Huang B, Wu H, Li ZM, Ye Q, Zhang YHP. Production of succinate from acetate by metabolically engineered Escherichia coli. ACS Synth Biol. 2016;5:1299-307.

24. Li ZJ, Shi ZY, Jian J, Guo YY, Wu Q, Chen GQ. Production of poly(3hydroxybutyrate-co-4-hydroxybutyrate) from unrelated carbon sources by metabolically engineered Escherichia coli. Metab Eng. 2010;12:352-9.

25. Spiekermann P, Rehm BHA, Kalscheuer R, Baumeister D, Steinbüchel A. A sensitive, viable-colony staining method using Nile red for direct screening of bacteria that accumulate polyhydroxyalkanoic acids and other lipid storage compounds. Arch Microbiol. 1999;171:73-80.

26. Kovach ME, Elzer PH, Hill DS, Robertson GT, Farris MA, Roop IIRM, Peterson KM. Four new derivatives of the broad-host-range cloning vector pBBR1MCS, carrying different antibiotic-resistance cassettes. Gene. 1995;166:175-6.

27. Li ZJ, Qiao KJ, Shi WC, Pereira B, Zhang HR, Olsen BD, Stephanopoulos G. Biosynthesis of poly (glycolate-co-lactate-co-3-hydroxybutyrate) from glucose by metabolically engineered Escherichia coli. Metab Eng. 2016:35:1-8.

28. Kumari S, Tishel R, Eisenbach M, Wolfe AJ. Cloning, characterization, and functional expression of acs, the gene which encodes acetyl coenzyme A synthetase in Escherichia coli. J Bacteriol. 1995;177:2878-86.

29. Wolfe AJ. The acetate switch. Microbiol Mol Biol Rev. 2005:69:12-50.
30. Li ZJ, Qiao KJ, Liu N, Stephanopoulos G. Engineering Yarrowia lipolytica for poly-3-hydroxybutyrate production. J Ind Microbiol Biotechnol. 2017:44:605-12.

31. Wang RY, Shi ZY, Chen JC, Wu Q, Chen GQ. Enhanced co-production of hydrogen and poly-(R)-3-hydroxybutyrate by recombinant PHB producing E. coli over-expressing hydrogenase 3 and acetyl-CoA synthetase. Metab Eng. 2012;14:496-503.

32. Li ZJ, Cai L, Wu Q, Chen GQ. Overexpression of NAD kinase in recombinant Escherichia coli harboring the phbCAB operon improves poly(3hydroxybutyrate) production. Appl Microbiol Biotechnol. 2009;83:939-47.

33. Huang B, Yang H, Fang G, Zhang X, Wu H, Li Z, Ye Q. Central pathway engineering for enhanced succinate biosynthesis from acetate in Escherichia coli. Biotechnol Bioeng. 2018;115:943-54.

34. Lee IY, Kim MK, Park YH, Lee SY. Regulatory effects of cellular nicotinamide nucleotides and enzyme activities on poly (3-hydroxybutyrate) synthesis in recombinant Escherichia coli. Biotechnol Bioeng. 1996;52:707-12.

35. Lv L, Ren YL, Chen JC, Wu Q, Chen GQ. Application of CRISPRi for prokaryotic metabolic engineering involving multiple genes, a case study: controllable P(3HB-co-4HB) biosynthesis. Metab Eng. 2015;29:160-8.

36. Wang $Y, W u H$, Jiang $X R$, Chen GQ. Engineering Escherichia coli for enhanced production of poly(3-hydroxybutyrate-co-4-hydroxybutyrate) in larger cellular space. Metab Eng. 2014;25:183-93.

37. Valentin HE, Dennis D. Production of poly(3-hydroxybutyrate-co4-hydroxybutyrate) in recombinant Escherichia coli grown on glucose. J Biotechnol. 1997;158:33-8.

38. Choi Jl, Sang YL. High-level production of poly(3-hydroxybutyrate-co3-hydroxyvalerate) by fed-batch culture of recombinant Escherichia coli. Appl Environ Microbiol. 1999;65:4363-8.

39. Chien CC, Li HH, Soo PC, Chen SY, Wei YH, Chen WM. Effects of different substrate composition on biosynthesis of polyhydroxybutyrate-cohydroxyvalerate by recombinant Escherichia coli. Appl Biochem Biotechnol. 2012;166:796-804

40. Liu XW, Wang HH, Chen JY, Liu XT, Chen GQ. Biosynthesis of poly(3hydroxybutyrate-co-3-hydroxyvalerate) by recombinant Escherichia coli harboring propionyl-CoA synthase gene ( $p r p E$ ) or propionate permease gene (prpP). Biochem Eng J. 2009;43:72-7.

41. Rivas B, Moldes AB, Dominguez JM, Parajo JC. Development of culture media containing spent yeast cells of Debaryomyces hansenii and corn steep liquor for lactic acid production with Lactobacillus rhamnosus. Int J Food Microbiol. 2004;97:93-8.

42. Liu BB, Yang MH, Qi BK, Chen XR, Su ZG, Wan YH. Optimizing I-(+)-lactic acid production by thermophile Lactobacillus plantarum As. 1.3 using alternative nitrogen sources with response surface method. Biochem Eng J. 2010;52:212-9.

43. Saxena J, Tanner RS. Optimization of a corn steep medium for production of ethanol from synthesis gas fermentation by Clostridium ragsdalei. World J Microbiol Biotechnol. 2012;28:1553-61.
Ready to submit your research? Choose BMC and benefit from:

- fast, convenient online submission

- thorough peer review by experienced researchers in your field

- rapid publication on acceptance

- support for research data, including large and complex data types

- gold Open Access which fosters wider collaboration and increased citations

- maximum visibility for your research: over $100 \mathrm{M}$ website views per year

At BMC, research is always in progress.

Learn more biomedcentral.com/submissions 